\title{
The Electrokinetic Cross-Coupling Coefficient: Two-Scale Homogenization Approach
}

\author{
Vladimir Shelukhin ${ }^{1}$, Igor Yeltsov ${ }^{2}$, Iliya Paranichev ${ }^{1}$ \\ ${ }^{1}$ Lavrentyev Institute of Hydrodynamics, Novosibirsk, Russia \\ ${ }^{2}$ Trofimuk Institute of Petroleum-Gas Geology and Geophysics, Novosibirsk, Russia \\ E-mail: Shelukhin@list.ru \\ Received April 7, 2011; revised May 9, 2011; accepted May 19, 2011
}

\begin{abstract}
By the two-scale homogenization approach we justify a two-scale model of ion transport through a layered membrane, with flows being driven by a pressure gradient and an external electrical field. By up-scaling, the electroosmotic flow equations in horizontal thin slits separated by thin solid layers are approximated by a homogenized system of macroscale equations in the form of the Poisson equation for induced vertical electrical field and Onsager's reciprocity relations between global fluxes (hydrodynamic and electric) and forces (horizontal pressure gradient and external electrical field). In addition, the two-scale approach provides macroscopic mobility coefficients in the Onsager relations. On this way, the cross-coupling kinetic coefficient is obtained in a form which does involves the $\zeta$-potential among the data provided the surface current is negligible.
\end{abstract}

Keywords: Electroosmosis, Two-Scale Homogenization, Cross-Coupling Coefficient

\section{Introduction}

In numerous studies on the electrolyte flows in rocks, the pore pressure $p$ and the streaming (electric) potential $\Psi$ interplay through the equation $\boldsymbol{j}=-L_{0} \nabla p-\sigma_{r} \nabla \Psi$, where $\boldsymbol{j}$ is the current density, $\sigma_{r}$ is the saturated rock conductivity, and $L_{0}$ is the electrokinetic crosscoupling term. Hydrogeological applications concern the study of water leakage from dams [1], groundwater flows in geothermal fields and volcanoes [2], estimation of water resources [3]. In electrochemistry, the above equation form a basis for managing microchip separations of analytes in nano-channels [4]; there is also an evidence that this equation find applications in hydrocarbon recovery $[5,6]$.

By the Helmholtz-Smoluchowski theory [7], the term $L_{0}$ is given by the formula $L_{0}=-\varphi \varepsilon_{f} \zeta / \mu$ where $\varphi$ is the porosity, $\varepsilon_{f}$ is the dielectric permittivity of the saturating fluid, $\mu$ is the viscosity, and $\zeta$ is the so-called $\zeta$-potential, the electric potential across the diffuse part of the interfacial double layer. In $[8,9,10]$, the above formula is substituted by $L_{0}=-\varepsilon_{f} \zeta /\left(\mu F_{0}\right)$ (or more sophisticated formulas), with $F_{0}$ being a dimensionless formation factor.

The goal of the present paper is to give more mathematical insight into the physico-chemical nature of the cross coupling coefficient $L_{0}$. Restricting ourselves to one-dimensional flows, we derive a representation formula for $L_{0}$ by the two-scale homogenization technique [11,12], starting from the equations of the ions transport through a layered membrane with a periodical structure. On this way we arrive at electro-osmotic macro-equations, whereas electrokinetic coupling coefficients can be determined from micro-equations defined on the periodicity cell.

Homogenization is a process in which the composite material with microscopic structure is replaced by an equivalent material with macroscopic, homogeneous properties. There are two methods of up-scaling coupled equations at the microscale to equations valid at macroscale for fluid-saturated porous media. The first is the volume averaging and the second is the two-scale and multiscale homogenization. Volume averaging has been applied successfully to derive the form of Biot's equations of poroelasticity [13], and a wide variety of other upscaling problems in double-porosity poroelasticity [14]. The averaging theorem used by all these authors is due to J. C. Slattery (1967) [15] and is based on well-known Green's theorem together with the idea that in relatively 
small regions volume averages of spatial gradient in statistically homogeneous media are presumably closely related to gradient of volume averages.

The two-scale homogenization method requires that the heterogeneous microstructure of a rock sample is described by spatially periodic parameters and the microscale of the heterogeneous porous medium is much smaller than the macroscale of most interest. The approach involves assuming that any quantity can be treated as a function of a macroscale variable and a microscale variable. The two-scale homogenization is a well established method in the theory of partial differential equations with rapidly oscillating periodic coefficients. This method has a lot of important applications in various branches of physics, mechanics and modern technology: porous media, composite and perforated materials, thermal conduction, acoustics, electromagnetism. For general references on the homogenization theory we refer to $[12,16,17,18]$.

The two-scale homogenization method can give formulas for coefficients in the up-scaled equations, whereas volume averaging methods give the form of the up-scaled equations but generally must be supplemented with physical arguments and/or data in order to determine the coefficients. A more detailed comparison of two up-scaling methods can be found in [19].

The present study is applicable to sandstones if surface conductivity can be neglected. When passing to claycontaining rocks one should also take into account bound charges concentrating on the interface surfaces. Such rocks are not considered here.

\section{Background}

Within the frame of the nonequilibrium thermodynamics, the fluxes (the Darcy's volume fluid velocity $\boldsymbol{q}$ and the electric current density $\boldsymbol{j}$ ) are derived as a linear combination of thermodynamical forces (the pressure gradient $p$ and the electric potential gradient $\Psi$ ):

$$
\begin{aligned}
\boldsymbol{q} & =-(k / \mu) \nabla p-L_{0} \nabla \Psi, \\
\boldsymbol{j} & =-L_{0} \nabla-\sigma_{r} \nabla \Psi,
\end{aligned}
$$

where $k$ is the permeability. Our goal is to show that these equations, specified for one-dimensional flows through a layered membrane, can be derived by the two-scale homogenization technique starting from the equations valid at microscale. While deriving the up-scaled Equations (1) and (2), (which can trace back to Helmholtz and von Smoluchowski) we obtain a formula for the cross-coupling coefficient $L_{0}$.

In this section, we summarize equations that govern the flows of a binary electrolyte solution through the pore space of a solid dielectric. To make clear our hypotheses on physical parameters, we use the Gaussian system of units. Clearly, while comparing final calculations with experiments, we apply the SI units. The electric field $\boldsymbol{E}$ obeys the charge conservation law

$$
\operatorname{div}\left(\varepsilon_{f} \boldsymbol{E}\right)=4 \pi q, \quad q=\sum_{i= \pm} q_{i} n_{i},
$$

where $\varepsilon_{f}$ is the fluid dielectric permittivity, $q_{+} \equiv$ $q_{1}(>0)$ is the charge of a positive ion, $q_{-} \equiv q_{2}(<0)$ is the charge of the negative ion, $n_{i}$ is the ion concentration. Viscous incompressible flows of the electrolyte solution is governed by the Navier-Stokes Equations [7]

$$
\mu \Delta \boldsymbol{v}-\nabla p=-q \boldsymbol{E}, \operatorname{div} \boldsymbol{v}=0
$$

with the inertial terms being neglected in the first momentum equation. Here, $\mathbf{v}$ is the velocity of the bulk fluid. The motion of both the ionic species satisfies the transport equation

$$
\frac{\partial n_{i}}{\partial t}+\operatorname{div} \boldsymbol{J}^{i}=0,
$$

with the flux given by the Nernst-Plank relation [7]

$$
\boldsymbol{J}^{i}=n_{i} \boldsymbol{v}-D^{i} \nabla n_{i}+\frac{D^{i} q_{i} n_{i}}{k_{B} T} \boldsymbol{E}
$$

where $D^{i}$ is the diffusion coefficient, $k_{B}$ is the Boltzmann constant, $T$ is the absolute temperature.

Inside the solid dielectric, the electrical field obeys the equation

$$
\operatorname{div}\left(\varepsilon_{s} \boldsymbol{E}\right)=0
$$

where $\varepsilon_{s}$ is the solid dielectric permittivity. In what follows, $\Psi$ stands for the electric potential, $\boldsymbol{E}=$ $-\nabla \Psi$.

The solid-fluid boundary conditions will be formulated below for one-dimensional flows.

\section{One-Dimensional Flows}

To motivate our further study we keep in mind a vertical membrane of thickness $l$ (see Figure 1) when the inflow pressure $p_{-}$(on the left) is grater than the outflow pressure $p_{+}$. It is the pressure gradient $\left(p_{+}-p_{-}\right) / l \equiv-\alpha$ which mainly controls the flow. It is also possible that the flow is due to the external electrical field $E=-\left(\psi^{+}-\psi^{-}\right) / l \equiv-\beta$. Commonly, an inflow concentration $n_{* i}$ of the $i$-th ion is prescribed on the left.

Now, to perform analytical study of the flow equations, we consider a vertical "membrane" of an infinite thickness. We study electrolyte steady flows through the horizontal layer of thickness $L$ consisting of $N$ horizontal thin slits $a_{m}<z<b_{m}$ of the same thickness $h_{f}$ separated by layers $b_{m}<z<a_{m+1}$ of a solid 
Z
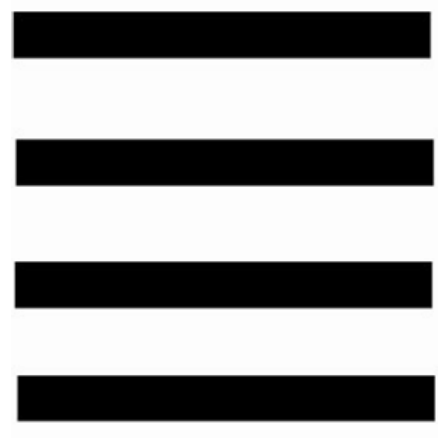

1

Figure 1. Layered membrane of the thicknes $I$ consisting of $N$ solid/liquid layers.

dielectric of the same thickness $h_{s}$. The central points $d_{m}$ of the liquid intervals $a_{m}<z<b_{m}$ are the points of reference where the ion inflow densities $n_{i}$ take the prescribed values $n_{* i}$.

Let $Q_{f}$ and $Q_{s}$ stand for fluid and solid domains

$$
\begin{gathered}
Q_{f}=\left\{x, z:-\infty<x<+\infty, z \in \Omega_{f}\right\}, \\
Q_{s}=\left\{x, z:-\infty<x<+\infty, z \in \Omega_{s}\right\}, \\
\Omega_{f}=\bigcup_{m=0}^{N-1}\left\{a_{m}<z<b_{m}\right\}, \Omega_{s}=\bigcup_{m=0}^{N-1}\left\{b_{m}<z<a_{m+1}\right\}, \\
a_{m}=m h, \quad b_{m}=a_{m}+h_{f}, \\
d_{m}=a_{m}+h_{f} / 2, h=h_{f}+h_{s} .
\end{gathered}
$$

In the domain $\Omega_{f}$, we look for steady solutions of the fluid Equations (3)-(5) in the form

$$
\begin{gathered}
\mathbf{v}=(v(z), 0,0)^{T}, p=\alpha x+P(z), \\
\Psi=\beta x+\varphi(z), \quad n_{i}=n_{i}(z),
\end{gathered}
$$

where $\alpha$ and $\beta$ are given constants. Under these assumptions, Equations (3)-(5) in each fluid interval $\left(a_{m}, b_{m}\right)$ become

$$
\begin{gathered}
\varepsilon_{f} \varphi_{z z}=-4 \pi q, \mu v_{z z}-\alpha-\beta q=0, \frac{\mathrm{d} J_{3}^{i}}{\mathrm{~d} z}=0, \\
J_{3}^{i}=-D^{i} n_{z}^{i}-\frac{D^{i} q_{i} n_{i}}{k_{B} T} \varphi_{z} .
\end{gathered}
$$

We study horizontal flows along the $x$-axis, hence $J_{3}^{i}=0$. The latter equality is equivalent to

$$
\frac{\mathrm{d}}{\mathrm{d} z}\left(\ln n_{i}+\frac{q_{i} \varphi}{k_{B} T}\right)=0 \text {. }
$$

Integrating between $d_{m}$ and $z \in\left(a_{m}, b_{m}\right)$, we exclude the concentration functions from consideration by the formula

$$
n_{i}(z)=n_{* i} \exp \left\{-\frac{q_{i}}{k_{B} T}\left[\varphi(z)-\varphi\left(d_{m}\right)\right]\right\} .
$$

In the solid intervals $b_{m}<z<a_{m+1}$, the potential $\varphi$ satisfies the equatio

$$
\varepsilon_{s} \varphi_{z z}=0 .
$$

In what follows we assume that the dielectric permittivity function and the fluid indicator function

$$
\varepsilon(z)=\left\{\begin{array}{ll}
\varepsilon_{f}, & z \in \Omega_{f}, \\
\varepsilon_{s}, & z \in \Omega_{s},
\end{array} \quad \mathbf{1}_{f}(z)= \begin{cases}1, & z \in \Omega_{f}, \\
0, & z \in \Omega_{s},\end{cases}\right.
$$

are extended periodically on the real line $\mathbb{R}$. Given a function $f(z)$ continuous everywhere except a point $z_{0}$, we introduce the jump as follows

$$
\left.[f]\right|_{z=z_{0}}=\lim _{\sigma \rightarrow 0}\left(f\left(z_{0}+\sigma\right)-f\left(z_{0}-\sigma\right)\right) .
$$

In some sandstones, surface conductivity can be neglected depending on the pore water salinity and the cation exchange capacity of the mineral surface. For such sandstones, the "electric" boundary conditions reduce to the conditions of continuity of the potential $\varphi$ and the normal component the electric induction vector $\varepsilon E$ :

$$
\left.[\varphi]\right|_{z=a_{k}, b_{l}}=\left.\left[\varepsilon \varphi_{z}\right]\right|_{z=a_{k}, b_{l}}=0,
$$

where $\mathrm{k}=1, \cdots, \mathrm{n}-1$ and $\mathrm{l}=0, \cdots, \mathrm{n}-1$.

The velocity satisfies the no-slip conditions

$$
\left.v\right|_{z=a_{k}, b_{k}}=0, \quad k=\overline{1, N-1} .
$$

We assume that $\varphi$ satisfies the external boundary conditions

$$
\left.\varphi\right|_{z=0}=\zeta_{0},\left.\quad \varphi\right|_{z=L}=\zeta_{L},
$$

with the prescribed $\zeta$-potentials $\zeta_{0}$ and $\zeta_{L}$. We introduce a function $[z]_{e}$, which takes the value of the integer part of the number $z$. Then the functions

$$
\begin{gathered}
H_{a}(z)=h\left[\frac{z}{h}\right]_{e}, \quad H_{d}(z)=\frac{h_{f}}{2}+h\left[\frac{z}{h}\right]_{e} \\
H_{b}(z)=h_{f}+h\left[\frac{z}{h}\right]_{e}
\end{gathered}
$$

take constant values $a_{m}, d_{m}, b_{m}$ for $a_{m}<z<a_{m+1}$. Thus to define $\varphi$ on the whole interval $0<z<L$, one should solve the non-local Poisson-Boltzmann equation

$$
\begin{aligned}
& \left(\varepsilon \varphi_{z}\right)_{z}= \\
& -4 \pi \mathbf{1}_{f}(z) \sum_{i} n_{* i} q_{i} \exp \left\{-\frac{q_{i}}{k_{B} T}\left[\varphi(z)-\varphi\left(H_{d}(z)\right)\right]\right\}
\end{aligned}
$$

jointly with the conditions (8) and (10). Observe that the 
function $\xi_{d}=H_{d}(z)-z$ is periodic, and $\xi_{d}=h_{f} / 2-z$ on the interval of periodicity $0<z<h$.

With the function $\varphi(z)$ at hand, one can find a velocity $v(z)$ from Equation (6) and the boundary conditions (9).

\section{Nondimensionalisation}

We look for an asymptotic solution of problem (11), (8), (10), (6), (9) for the functions $\varphi_{z^{\prime} z^{\prime}}^{\prime}=0$. and $v(z)$, assuming that the ratio $h / L=1 / N=\delta$ is a small parameter for some positive entire number $N$. We argue by the homogenization approach [24], so the entire interval $\Omega=\{0<z<L\}$ is fixed and $\delta$ varies in $(0,1)$. In that case $h=\delta L$ and

$$
h_{f}=\delta \bar{h}_{f}, h_{s}=\delta \bar{h}_{s}, \bar{h}_{f}+\bar{h}_{s}=L, \phi:=\bar{h}_{f} / L
$$

Here, $\phi$ is the porosity.

We call $z \in \Omega$ a slow variable and we introduce the fast variable $y=z /(\delta L)$. With $\delta$ being small, the periodic functions $\varepsilon(z)$ and $\mathbf{1}_{f}(z)$ oscillate strongly and they can be represented as functions of the fast variable:

$$
\varepsilon(z)=\tilde{\varepsilon}\left(\frac{z}{\delta L}\right), \quad \mathbf{1}_{f}(z)=\tilde{\mathbf{1}}_{f}\left(\frac{z}{\delta L}\right),
$$

where

$$
\tilde{\varepsilon}(y)=\left\{\begin{array}{l}
\varepsilon_{f}, 0<y<\phi, \\
\varepsilon_{s}, \phi<y<1,
\end{array} \quad \tilde{\mathbf{1}}_{f}(y)=\left\{\begin{array}{l}
1,0<y<\phi, \\
0, \phi<y<1,
\end{array}\right.\right.
$$

are periodic functions with the period equal to 1 . In what follows the functions

$$
\tilde{\xi}_{a}(y)=-L y, \quad \tilde{\xi}_{d}(y)=L(\phi / 2-y)
$$

and

$$
\tilde{\xi}_{b}(y)=L(\phi-y), \quad 0<y<1,
$$

are extended periodically for all $y \in \mathbb{R}$. The functions $H_{a}(z), H_{d}(z), H_{b}(z)$ can be written as

$$
H_{a}(z)=z+\delta \tilde{\xi}_{a}\left(\frac{z}{\delta L}\right), \quad H_{d}(z)=z+\delta \tilde{\xi}_{d}\left(\frac{z}{\delta L}\right),
$$

and

$$
H_{b}(z)=z+\delta \tilde{\xi}_{b}\left(\frac{z}{\delta L}\right)
$$

In the notations used, the function $\varphi(z)$ on the interval $0<z<L$ is a solution of the problem

$$
\begin{gathered}
\frac{d}{d z}\left(\tilde{\varepsilon}\left(\frac{z}{\delta L}\right) \frac{d}{d z} \varphi\right)=-4 \pi \tilde{\mathbf{1}}_{f}\left(\frac{z}{\delta L}\right) F[\varphi, z], \\
\left.\varphi\right|_{z=0}=\zeta_{0},\left.\quad \varphi\right|_{z=L}=\zeta_{L},
\end{gathered}
$$

where $F[\varphi, z]$ is equal to

$$
\sum_{i} n_{* i} q_{i} \exp \left\{-\frac{q_{i}}{k_{B} T}\left[\varphi(z)-\varphi\left(z+\delta \tilde{\xi}_{d}\left(\frac{z}{\delta L}\right)\right)\right]\right\}
$$

It follows from Equations (6) that the bulk velocity satisfies the equation

$$
\mu v_{z z}+\frac{\beta \varepsilon_{f}}{4 \pi} \varphi_{z z}=\alpha .
$$

Let us perform scaling, using the symbol $\bar{a}$ for a reference value of the dimensional quantity $a$ and the symbol $a^{\prime}$ for a dimensionless quantity of $a$, i. e. $a=\bar{a} a^{\prime}$. We use the following notations:

$$
\begin{gathered}
z=L z^{\prime}, \quad x=L x^{\prime}, \quad n_{i}=\bar{n} n_{i^{\prime}}, \quad q_{i}=\bar{q} q_{i^{\prime}} \\
\bar{\varphi} \varphi^{\prime}\left(z^{\prime}\right)=\left.\varphi(z)\right|_{z=L z^{\prime}}, \quad v=\bar{v} v^{\prime} \\
\alpha=\frac{\bar{p} \alpha^{\prime}}{L} \equiv \frac{\bar{p} p_{x^{\prime}}}{L}, \quad \beta=\frac{\bar{\varphi} \beta^{\prime}}{L} \equiv \frac{\bar{\varphi} \Psi_{x^{\prime}}}{L}, \\
H_{d}(z)=L H_{d^{\prime}}\left(z^{\prime}\right), \quad D_{i}=\bar{D} D^{\prime} .
\end{gathered}
$$

The quantity

$$
l_{d}=\sqrt{\frac{\varepsilon_{f} k_{B} T}{2 \overline{n q}^{2}}}
$$

is known as the Debye length. In terms of dimensionless variables Equations (13) and (11) in the fluid domain $\Omega_{f}^{\prime}=\cup_{m=0}^{N-1}\left\{a_{m}^{\prime}<z^{\prime}<b_{m}^{\prime}\right\}$ take the form

$$
A_{4} v_{z^{\prime} z^{\prime}}^{\prime}+\frac{A_{1} A_{2} A_{3} \beta^{\prime}}{2 \pi} \varphi_{z^{\prime} z^{\prime}}^{\prime}=\alpha^{\prime}
$$

$$
\begin{aligned}
& A_{1} A_{2} \varphi_{z^{\prime} z^{\prime}}^{\prime} \\
& =-2 \pi \sum_{i} n_{* i} q_{i}^{\prime} \exp \left\{q_{i}^{\prime} A_{1}\left[\varphi^{\prime}\left(H_{d^{\prime}}\left(z^{\prime}\right)\right)-\varphi^{\prime}\left(z^{\prime}\right)\right]\right\} .,
\end{aligned}
$$

Here,

$$
A_{1}=\frac{\bar{q} \bar{\varphi}}{k_{B} T}, A_{2}=\frac{l_{d}^{2}}{L^{2}}, A_{3}=\frac{\bar{q} \bar{\varphi} \bar{n}}{\bar{p}}, A_{4}=\frac{\mu \overline{v n}}{\bar{p}} .
$$

In the solid domain Equation (7) becomes $\varphi_{z^{\prime} z^{\prime}}^{\prime}=0$.

Assuming that the dimensionless quantities $A_{i}$ satisfy the equalities

$$
A_{i}=O\left(\delta^{n_{i}}\right), \quad i=1, \cdots 4, \quad \text { as } \quad \delta \rightarrow 0,
$$

we obtain a hierarchy of problems to study. In this paper we restrict ourselves to the case when all the powers $n_{i}$ are equal to zero, i. e. $A_{i}=O(1)$. The meaning of these hypotheses is the following. The relation $A_{1}=O(1)$ implies that electroosmotic force and thermal force are of the same order. Observe that the relation $A_{1}=O(1)$ holds, for example, for the symmetric electrolyte (where $\left|q_{+}\right|=\left|q_{-}\right|$and $\left.n_{*+}=n_{*-}\right)$ in water at $T=298 \mathrm{~K}$, with the valency $z=1$ and with the $\zeta$-potential equal to $25 \mathrm{mV}$ [4]. When $A_{1}$ is not small, the Debye- 
Hückel linearization of the Poisson-Boltzmann equation does not work. Under the condition $A_{1}=O(1)$ the Debye length $l_{d}$ can be longer compared to electrical double layer, moreover the double layer overlapping could occur. Indeed, it is a useful rule of thumb that $l_{d}=9.6 /(z \sqrt{\bar{n}})$ [4] where $z$ is the valency. For the above mentioned electrolyte with the counterion molar concentration $\bar{n}=0,01 \mathrm{mM}$ we have $l_{d}=100 \mathrm{~nm}$, whereas the double electric layer is normally only a few nanometers thick [4] and the nanocapillary membrane may have the pore diameter of $15 \mathrm{~nm}$ [20]. For such cases the hypothesis $A_{2}=O(1)$ is natural. Hypothesis $A_{3}=O(1)$ amounts to the effect that the horizontal pressure gradient and the applied horizontal electrical field are of the same order. The relation $A_{4}=O(1)$ means that viscous response is of the same order as the applied horizontal pressure gradient.

There is one more assumption that we impose on the Péclet number $P e=\overline{v L} / \bar{D}$ :

$$
P e=O(1) \quad \text { as } \quad \delta \rightarrow 0 .
$$

The hypothesis implies that convection and diffusion are of the same order.

We close this section by reminding the Debye-Hückel approach to the Poisson-Boltzmann Equation (11) in the single layer $z>0$ with the boundary conditions $\varphi \rightarrow 0$ and $\varphi_{z} \rightarrow 0$ as $z \rightarrow \infty$ and $\left.\varphi\right|_{z=0}=\zeta_{0}$. In the case of symmetric electrolyte, the linearized equation (11), in the SI system of units where $4 \pi$ is substituted by 1 , becomes $l_{d}^{2} \varphi_{z z}=-\varphi$, since the nonlocal term $\varphi(d)$ vanishes as $d \rightarrow \infty$. Clearly, $\varphi=\zeta_{0} e^{-z / l_{d}}$ is a solution. This explains the notion (14).

\section{Asymptotic Analysis of Electric Field}

We proceed by returning to the dimensional variables. Using the method of the two-scale expansions [12], we look for the solution of Equation (12) in the form of an expansion series

$$
\varphi_{\delta}(z)=\left.\sum_{0}^{\infty} \delta^{k} \varphi^{k}(z, y)\right|_{y=z /(\delta L)},
$$

where the functions $\varphi^{k}(z, y)$ are periodic in the variable $y, 0<y<1$, with a period equal to 1 for each $z \in \Omega$. We introduce the flux

$$
S_{\delta}(z) \equiv \tilde{\varepsilon}\left(\frac{z}{\delta L}\right) \frac{\mathrm{d}}{\mathrm{d} z} \varphi_{\delta}(z) .
$$

Clearly,

$$
\frac{\mathrm{d}}{\mathrm{d} z} S_{\delta}=-4 \pi \tilde{\mathbf{1}}_{f} F\left[\varphi_{\delta}, z\right],
$$

We present this flux as a series

$$
S_{\delta}(z)=\left.\sum_{0}^{\infty} \delta^{k} S^{k}(z, y)\right|_{y=z /(\delta L)},
$$

where the functions $S^{k}(z, y)$ are 1-periodic in $y$ for all $z \in \Omega$.

Using the formula

$$
\frac{\mathrm{d}}{\mathrm{d} z} \varphi^{k}\left(z, \frac{z}{\delta L}\right)=\varphi_{z}^{k}\left(z, \frac{z}{\delta L}\right)+\frac{1}{\delta L} \varphi_{y}^{k}\left(z, \frac{z}{\delta L}\right),
$$

and substituting the series (16) and (19) into equality (17), we obtain an equality which looks like

$$
\sum_{-1}^{\infty} \delta^{k}(\ldots)_{k}=0 .
$$

Thus $(\ldots)_{k}=0$ for all $k=-1,0,1, \cdots$. In particular, the three first equalities can be written as

$$
\varphi_{y}^{0}(z, y)=0
$$

and

$$
\begin{aligned}
& S^{0}(z, y)=\tilde{\varepsilon}(y)\left(\varphi_{z}^{0}(z, y)+\frac{\varphi_{y}^{1}(z, y)}{L}\right), \\
& S^{1}(z, y)=\tilde{\varepsilon}(y)\left(\varphi_{z}^{1}(z, y)+\frac{\varphi_{y}^{2}(z, y)}{L}\right) .
\end{aligned}
$$

Substituting the series (16) and (19) into equality (18) and paying attention to the powers $\delta^{-1}$ and $\delta^{0}$, we obtain the equations

$$
\begin{gathered}
\frac{\partial}{\partial y}\left\{\tilde{\varepsilon}(y)\left[\varphi_{z}^{0}(z, y)+L^{-1} \varphi_{y}^{1}(z, y)\right]\right\}=0 \\
\frac{\partial}{\partial z}\left\{\tilde{\varepsilon}(y)\left[\varphi_{z}^{0}(z, y)+L^{-1} \varphi_{y}^{1}(z, y)\right]\right\}+, \\
L^{-1} \frac{\partial}{\partial y}\left\{\tilde{\varepsilon}(y)\left[\varphi_{z}^{1}(z, y)+L^{-1} \varphi_{y}^{2}(z, y)\right]\right\}= \\
=-4 \pi \tilde{\mathbf{1}}_{f}(y) \sum_{i} q_{i} n_{* i} .
\end{gathered}
$$

Equations (20) and (21) allow one to determine the functions $\phi^{0}(z, y), \phi^{1}(z, y)$ and $\phi^{2}(z, y)$ uniquely. Indeed, with a function $\phi^{0}$ independent of the variable $y$, we look for $\phi^{1}(z, y)$ by the method of separation of variables assuming that there exists a 1 -periodic function $\omega_{1}(y)$ such that

$$
\varphi^{1}(z, y)=\varphi_{z}^{0}(z) \omega_{1}(y) .
$$

Substituting this presentation into Equation (20), we find that the function $\omega_{1}(y)$ solves the following problem on the interval $0<y<1$ :

$$
\frac{\mathrm{d}}{\mathrm{d} y}\left(\tilde{\varepsilon}(y)\left(1+\frac{1}{L} \frac{\mathrm{d} w_{1}}{\mathrm{~d} y}\right)\right)=0, \quad \int_{0}^{1} w_{1}(y) \mathrm{d} y=0 .
$$

The latter integral condition serves for uniqueness. We 
integrate and arrive at the formulas

$$
\begin{gathered}
\tilde{\varepsilon}(y)\left(1+\frac{1}{L} \frac{\mathrm{d} w_{1}}{\mathrm{~d} y}\right)=\varepsilon_{h}, \\
\varepsilon_{h}=\left(\int_{0}^{1} 1 / \tilde{\varepsilon}(y) \mathrm{d} y\right)^{-1}=\frac{1}{\phi / \varepsilon_{f}+(1-\phi) / \varepsilon_{s}} .
\end{gathered}
$$

Next, we use periodicity and integrate Equation (21) with respect to $y$ to obtain the following macroequation for $\varphi^{0}(z)$ :

$$
\varepsilon_{h} \varphi_{z z}^{0}=-4 \pi \phi \sum_{i} q_{i} n_{* i}
$$

As for the function $\varphi^{2}(z, y)$, we look it in the form $\varphi^{2}(z, y)=\varphi_{z z}^{0}(z) \omega_{2}(y)$.

Substituting this presentation into Equation (21), we find that $\omega_{2}(y)$ is a periodic solution of the problem

$$
\begin{aligned}
\varepsilon_{h} \varphi_{z z}^{0}+\frac{1}{L} \varphi_{z z}^{0} \frac{\mathrm{d}}{\mathrm{d} y}\left\{\tilde{\varepsilon}(y)\left(\omega_{1}(y)+\frac{1}{L} \frac{\mathrm{d}}{\mathrm{d} y} \omega_{2}(y)\right)\right\}= \\
-4 \pi \tilde{\mathbf{1}}_{f}(y) \sum_{i} q_{i} n_{* i} .
\end{aligned}
$$

This problem has a unique solution provided

$$
\int_{0}^{1} \omega_{2} \mathrm{~d} y=0
$$

Thus, we have established the following asymptotic equality for the electric potential:

$$
\varphi_{\delta}(z)=\varphi^{0}(z)+\delta \varphi^{1}\left(z, \frac{z}{\delta L}\right)+\delta^{2} \varphi^{2}\left(z, \frac{z}{\delta L}\right)+\cdots
$$

\section{Asymptotic Analysis of Velocity}

Integrating Equation (13), we obtain the following formula for velocity in each fluid domain $a_{m}<z<b_{m}$ :

$$
\mu \nu(z)=\frac{1}{b_{n}-a_{n}} \int_{a_{n}}^{Z} \mathrm{~d} r \int_{a_{n}}^{b_{n}} \mathrm{~d} s \int_{S}^{r} G(\lambda) \mathrm{d} \lambda,
$$

where

$$
G=\alpha-\frac{\beta \varepsilon_{f}}{4 \pi} \varphi_{\mathrm{zz}}
$$

We extent the function $v$ by zero to the solid intervals and denote such an extension by $\hat{v}_{\delta}(z)$. Now, with $L_{\delta}$ standing for $\delta L$, we have for all $z \in \Omega$ that

$$
\begin{aligned}
& \mu \hat{v}_{\delta}(z)= \\
& \frac{\mathbf{1}_{f}\left(z / L_{\delta}\right)}{\delta \bar{h}_{f}} \int_{z+\delta \tilde{\xi}_{a}\left(z / L_{\delta}\right)}^{z} \mathrm{z}+\delta \tilde{\xi}_{a}\left(z / L_{\delta}\right) \\
& z+\delta \tilde{\xi}_{b}\left(z / L_{\delta}\right) \\
& r
\end{aligned} \int_{S} G(\lambda) \mathrm{d} \lambda
$$

With $\varphi(z)$ given by the expansion series (16), we look for $\hat{v}_{\delta}(z)$ in the form

$$
\hat{v}_{\delta}(z)=\left.\sum_{2}^{\infty} \delta^{k} v^{k-2}(z, y)\right|_{y=Z / L_{\delta}},
$$

where the functions $v^{k}(z, y)$ are 1 -periodic in $y$ and $v^{k}(z, y)=0$ for $\varphi<y<1-\varphi$. After simple calculations, we find that

$$
\begin{gathered}
\int_{Z+\delta \tilde{\xi}_{a}\left(z / L_{\delta}\right)}^{Z} \mathrm{~d} r \int_{Z+\delta \tilde{\xi}_{a}\left(z / L_{\delta}\right)}^{Z+\delta \tilde{\xi}_{b}\left(z / L_{\delta}\right)} \mathrm{d} s \int_{S}^{r} \alpha \mathrm{d} \lambda= \\
\frac{\delta^{3} \alpha \bar{h}_{f}}{2} \tilde{\xi}_{a}\left(\frac{Z}{\delta L}\right) \tilde{\xi}_{b}\left(\frac{Z}{\delta L}\right) .
\end{gathered}
$$

Using the properties of functions $\varphi^{0}(z, y), \varphi^{1}(z, y)$, $\varphi^{2}(z, y)$, we obtain that $\varphi(\lambda)$ is equal to

$$
\varphi^{0}(z)+\delta^{1} \varphi_{z}^{0}(z) \omega_{1}(y)+\delta^{2} \varphi_{z z}^{0}(z) \omega_{2}(y)+\cdots,
$$

for $z=\lambda, \quad y=\lambda / L_{\delta}$

As for $\varphi^{\prime \prime}(\lambda)$, we find that it is equal to

$$
\left\{\frac{\varphi_{z}^{0} \omega_{1}^{\prime \prime}(y)}{\delta L^{2}}+\varphi_{z z}^{0}\left(1+\frac{2 \omega_{1}^{\prime}(y)}{L}+\frac{\omega_{2}^{\prime \prime}(y)}{L^{2}}\right)\right\}+\delta(\ldots) \text {. }
$$

for $z=\lambda, \quad y=\lambda / L_{\delta}$

By virtue of the multiplier $\mathbf{1}_{f}(z)$ in the right side of Equation (11), we can assume that $z \in\left(H_{a}(z), H_{b}(z)\right)$

Then, the variables $r, s$ belong to the interval

$$
\left(H_{a}(z), H_{b}(z)\right)
$$

also. As $\lambda$ is between $r$ and $s$, the inequalities

$$
0<\frac{\lambda}{\delta L}-\left[\frac{\lambda}{\delta L}\right]_{e}<\phi
$$

hold and the second derivatives of $\omega_{1}(\lambda), \omega_{2}(\lambda)$ in Equation (25) are meaningful. In addition, it follows from Equations (23), (24) and (25) that, for $0<y<\phi$, the functions $\omega_{1}(y), \omega_{2}(y)$ satisfy the equations

$$
\omega_{1} "(y)=0
$$

$$
\varphi_{\mathrm{zz}}^{0} \varepsilon_{f}\left(1+\frac{2}{L} \omega_{1} "(y)+\frac{1}{L^{2}} \omega_{2} "(y)\right)=-4 \pi \sum_{ \pm} q_{i} n_{* i} .
$$

Thus, we obtain

$$
\varphi^{\prime \prime}(\lambda)=-\frac{4 \pi}{\varepsilon_{f}} \sum_{i} q_{i} n_{* i}+\delta(\ldots) .
$$

Substituting Equations (28) and (29) into Equation (27) and considering only the power $\delta^{2}$, one can show that the function $v^{0}(z, y)$ does not depend on the variable $z$ and has the form

$$
\mu v^{0}(y)=\frac{1}{2} \mathbf{1}_{f}(y) \tilde{\xi}_{a}(y) \tilde{\xi}_{b}(y)\left(\alpha+\beta \sum_{i} q_{i} n_{* i}\right) .
$$

Integrating Equation (30) over the periodicity cell, we 
obtain the macroscopic equation

$$
V \equiv \int_{0}^{1} v^{0}(y) d y=-\lambda_{11} \alpha-\lambda_{12} \beta,
$$

where the hydrodynamic and electrochemical mobilities are defined by the formulas

$$
\lambda_{11}=\frac{L^{2} \phi^{3}}{12 \mu}, \quad \lambda_{12}=\frac{L^{2} \phi^{3}}{12 \mu} \sum_{i} q_{i} n_{* i} .
$$

Thus, we have established the following asymptotic equality for the velocity field:

$$
\hat{v}_{\delta}(z)=\delta^{2} V+o\left(\delta^{3}\right), \quad \text { as } \quad \delta \rightarrow 0 .
$$

We introduce the total electric current $\sum_{i} \mathbf{J}^{i} q_{i}$ whose horizontal component in the fluid phase is equal to

$$
J=v \sum_{i} q_{i} n_{i}-\frac{\beta}{k_{B} T} \sum_{i} q_{i}^{2} n_{i} D_{i} .
$$

We extent the function $J$ by zero to the solid intervals and denote such an extension by $\hat{J}_{\delta}(z)$. Due to hypothesis (15), we have that $D_{i}=\delta^{2} D_{* i}$. This is why we look for $\hat{J}_{\delta}(z)$ in the form of the expansion series

$$
\hat{J}_{\delta}(z)=\left.\sum_{k=2}^{\infty} \delta^{k} J^{k-2}(z, y)\right|_{y=z / L_{\delta}} .
$$

It follows from (32) and (28) that

$$
J^{0}(z, y)=v^{0}(y) \sum_{i} q_{i} n_{* i}-\frac{\beta}{k_{B} T} \sum_{i} q_{i}^{2} n_{* i} D_{* i} .
$$

By integration, we arrive at the macroscopic equation

$$
\bar{J} \equiv \int_{0}^{1} J^{0}(y) \mathrm{d} y=-\lambda_{21} \alpha-\lambda_{22} \beta,
$$

where $\lambda_{12}=\lambda_{21}$ and

$$
\lambda_{22}=\frac{L^{2} \phi^{3}}{12 \mu}\left(\sum_{i} q_{i} n_{* i}\right)^{2}+\frac{1}{k_{B} T} \sum_{i} q_{i}^{2} n_{* i} D_{* i} .
$$

Thus, we have established the following asymptotic equality for the electric current:

$$
\hat{J}_{\delta}(z)=\delta^{2} \bar{J}+o\left(\delta^{3}\right), \quad \text { as } \quad \delta \rightarrow 0 .
$$

The asymptotic equalities (26), (31) and (33) are valid in the sense of weak or two-scale convergences; mathematical aspects of these asymptotic expansions are extensively investigated in [21,22,23].

\section{Electrokinetic Coupling Coefficients}

We introduce the Darcy volumetric flow rate $q=\delta^{2} V$ and the current density $j=\delta^{2} \bar{J}$. By the above asymptotic analysis we have derived the macroequations (which are valid up to terms $o\left(\delta^{3}\right), \delta=h / L$ )

$$
\begin{aligned}
q & =-L_{11} p_{x}-L_{12} \Psi_{x}, j=-L_{21} p_{x}-L_{22} \Psi_{x}, \\
\varepsilon_{h} \Psi_{z z} & =-4 \pi \phi \sum_{i} q_{i} n_{* i},\left.\quad \Psi\right|_{z=0}=\zeta_{0},\left.\quad \Psi\right|_{z=L}=\zeta_{L},
\end{aligned}
$$

which describe electrolyte flow and distribution of the electric potential $\Psi$ across a layered membrane under the assumption that

$$
p_{x}, \quad \Psi_{x}, \quad n_{* i}, \quad \varsigma_{0}, \varsigma_{L}
$$

are prescribed data. For such a membrane, the effective dielectric permittivity $\varepsilon_{h}$ and the electrokine-

tic coupling coefficients $L_{k l}=\delta^{2} \lambda_{k l}$ are given by the formulas

$$
\begin{gathered}
1 / \varepsilon_{h}=\phi / \varepsilon_{f}+(1-\phi) / \varepsilon_{s}, \\
L_{11}=\frac{h_{f}^{2} \phi}{12 \mu}, \quad L_{12}=L_{21}=\frac{h_{f}^{2} \phi}{12 \mu} \sum_{i} q_{i} n_{* i}, \\
L_{22}=\frac{h_{f}^{2} \phi}{12 \mu}\left(\sum_{i} q_{i} n_{* i}\right)^{2}+\frac{1}{k_{B} T} \sum_{i} q_{i}^{2} n_{* i} D_{i} .
\end{gathered}
$$

Formula (35) stating that the effective permittivity $\varepsilon_{h}$ of the layered membrane is the harmonic mean of $\varepsilon_{s}$ and $\varepsilon_{f}$ was first derived by Maxwell (Maxwell 1881) in a different way [24]. Observe, that the Onsager reciprocity relation $L_{12}=L_{21}$ [25] is not imposed but derived in the above calculations as a consequence of the homogenization procedure. Moreover, the inequality

$$
L_{12}^{2}-L_{11} L_{22} \geq 0 \text {, }
$$

providing nonnegativity of the entropy production rate is also satisfied automatically [26] due to the representation formulas (36) and (37). The inequality (38) becomes equality if both the diffusion coefficients $D_{i}$ are negligible. Observe that for some free solutions $D_{i}=2 \cdot 10^{-9} \mathrm{~m}^{2} \mathrm{~s}^{-1}$ [27].

We emphasize that the coupling coefficients $L_{k l}$ in the macro-equations (34) are given by the representation formulas (36) and (37) as a result of an extensive analysis of the micro-Equations (22) and (25) for the functions $\omega_{1}(y)$ and $\omega_{2}(y)$ defined on the periodicity cell.

Clearly, the electroosmosis Equations (1) and (2) should coincide with the system (34) for one- dimensional flows. Whereas the formula

$$
L_{0}=-\phi \varepsilon_{f} \zeta / \mu
$$

for the cross-coupling coefficient have a drawback of measuring the $\zeta$-potential, the kinetic coefficients $L_{k l}$ derived by homogenization for the ideal (layered) porous medium do not depend on $\zeta$. One can exploit this advantage in calculation of the coupling coefficient $L_{0}$ 
for general porous media.

Applying the general Equations (1) and (2) to the ideal (layered) porous medium, we find that

$$
\begin{gathered}
\frac{k}{\mu}=L_{11}=\frac{h_{f}^{2} \phi}{12 \mu}, \quad L_{0}=L_{12}=\frac{h_{f}^{2} \phi}{12 \mu} \sum_{i} q_{i} n_{* i}, \\
\sigma_{r}=L_{22}=\frac{h_{f}^{2} \phi}{12 \mu}\left(\sum_{i} q_{i} n_{* i}\right)^{2}+\frac{1}{k_{B} T} \sum_{i} q_{i}^{2} n_{* i} D_{i} .
\end{gathered}
$$

Now, we have

$$
\sigma_{r}=\frac{L_{0}^{2} \mu}{k}+\frac{1}{k_{B} T} \sum_{i} q_{i}^{2} n_{* i} D_{i}
$$

and inequality (38) gives the following estimate for the electrokinetic cross-coupling coefficient $L_{0}$ :

$$
L_{0} \leq \sqrt{\sigma_{r} k / \mu} \text {. }
$$

As for real rocks, formulas (39) and (40) suggest to take $L_{0}$ in the form

$$
L_{0}=F \sqrt{\sigma_{r} k / \mu},
$$

where $F$ is a dimensionless geometrical factor. In applications, the above formula can be of use if no data are available for the diffusion coefficients $D_{i}$ and the $\zeta$ - potential. We emphasize that formula (41) is not a physical law but rather an engineering formula which can be of help for some sandstones when surface conductivity can be neglected.

Firstly, we evaluate $F$ for a rock sample on the basis of the F. F. Reuss experiment [28]. Such an experiment reveals that a difference in the electric potentials $\Delta_{\psi}$ applied to water in a U-tube results in a change of water levels when the low part of tube is plugged with a sandstone sample (Figure 2).

We calculate the weight $P$ of salt water which fills the cylinder of height $h$ with cross section area $S$ (Figure 2). We have $P=\rho_{f}$ shg, where $g$ is the

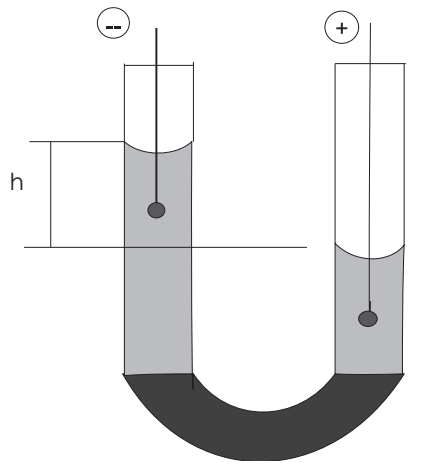

Figure 2. F. F. Reus experiment (1808) with the U-tube plugged with sandstone sample: applied electric field results in water level change of height $h$. gravitational acceleration and $\rho_{f}$ is the water density. The pressure drop across the sandstone plug is equal to $\Delta_{p}=\rho h g$. On the other hand it follows from Equations (34) that at equilibrium, when $q=0$, we have $\Delta_{p}=\Delta_{\psi} L_{12} / L_{11}=\Delta_{\psi} L_{0} \mu / k$.

In $[29,30]$, a mathematical model (jointly with a computer code) is developed for calculation of the electric conductivity $\sigma_{r}$ of a saturated rock. The model allows one to find an optimal Archie-like law

$$
\sigma_{r}=\sigma_{f}\left(\frac{\phi-\phi_{p}}{1-\phi_{p}}\right)^{m},
$$

where $\sigma_{f}$ is the conductivity of the saturating fluid, $\phi_{p}$ is the percolation threshold porosity, $m$ is the cementation factor. For sandstones, it was calculated in [29] that $\phi_{p}=0.03, m=1.5$. Thus, for sandstones, formula (41) becomes

$$
L_{0}=F\left(\frac{\phi-\phi_{p}}{1-\phi_{p}}\right)^{m / 2}\left(\frac{\sigma_{f} k}{\mu}\right)^{1 / 2}
$$

Now, the factor $F$ can be evaluated from the formula

$$
\frac{h}{\Delta_{\psi}}=\frac{F}{g \rho_{f}} \sqrt{\frac{\mu \sigma_{f}}{k}}\left(\frac{\phi-\phi_{p}}{1-\phi_{p}}\right)^{m / 2} .
$$

We perform calculation assuming that, as in [31], the applied potential difference $\Delta_{\psi}=100 \mathrm{~V}$ results in the water level difference $h=20 \mathrm{~cm}$. The rock data are taken from [6]: $\mu=1.05 \mathrm{cp}, k=250 \mathrm{mD}, \sigma_{f}=0.25 \mathrm{~S} / \mathrm{m}$, $\rho_{f}=0.93 \mathrm{~g} / \mathrm{cm}^{3}, \phi=0.36$. With these data at hand, we find that $F=1.25 \cdot 10^{-3}$. It is the cross-coupling coefficient $C=L_{0} / \sigma_{r}\left[V \mathrm{~Pa}^{-1}\right]$ that can be measured in applications [32]. With the factor $F$ given above, we find that $C=0.86 \cdot 10^{-7} \mathrm{~V} / \mathrm{Pa}$ in agreement with the data in [6].

Next, we calculate the factor $F$ for the rock sample composed of Berea sandstone 500 starting from experimental measurements of streaming potential when a fluid, with a prescribed $\mathrm{NaCl}$ concentration (500 ppm), flows through the sample [5]. Given data $\mu=1.05 \mathrm{cp}$, $\sigma_{r}=67.9 \cdot 10^{-4} \mathrm{~S} / \mathrm{m}, k=200 \mathrm{mD}, L_{0} / \sigma_{r}=0.17 \mu \mathrm{V} / \mathrm{Pa}$, we find from formula (41) that $F=1.02 \cdot 10^{-3}$.

\section{Conclusions}

We have proposed a two-scale model for one-dimensional horizontal electroosmotic flows in a number of thin horizontal slits, with a horizontal pressure gradient and a horizontal electrical field being driving forces. The model is derived within the framework of homogenization in the up-scaling of the pore-scale description 
consisting of Stokes equation for bulk fluid flow and the Nernst-Plunk equation for the ion transport. The homogenized model is a generalization both of the Darcy law and the Ohm low. According to this model, both the fluid flux and the electric flux depend linearly on the horizontal pressure gradient and the horizontal electrical field, with the coupling coefficients obeying the Onsager symmetry condition and not depending on the $\zeta$ potential.

As for three-dimensional general flows in sandstones in the case when surface current is negligible, the cross-coupling coefficient $L_{0}$ is obtained in the approximate form $L_{0}=F \sqrt{\sigma_{r} k / \mu}$, where $\sigma_{r}$ is the fluid saturated rock electric conductivity, $k$ is the rock permeability, $\mu$ is the fluid viscosity, and $F$ is dimensionless geometrical factor which depends on the sample. We evaluated that $F=1.02 \cdot 10^{-3}$ for Berea sandstones.

\section{Acknowledgements}

The authors were supported by Russian Fund of Fundamental Researches (grant 10-05-00835-a) and State Contract No. 14.740.11.0355 of Federal Special-Purpose Program "Personal".

\section{References}

[1] A. Bolève, A. Crespy, A. Revil, F. Janod and J. I. Mattiuzzo, "Streaming Potentials of Granular Media: Influencies of the Dukhin and Reynolds Numbers," Journal of Geophysical Research, Vol. 112, No. B8, 2007, pp. 1-14.

[2] A. Jardani, A. Revil, A. Bolève and J. P. Dupont, "The Three-Dimensional Inversion of Self-Potential Data Used to Constrain the Pattern of Groundwater Flow in Geothermal Fields," Journal of Geophysical Research, Vol. 113, No. 89, 2008, pp. 1-22.

[3] G. de Marsily, “Quantative Hydrology,” Academic Press, London, 1986.

[4] B. J. Kirby and E. F. Hasselbrink Jr., "Zeta Potential of Microfluidic Substrates. 1. Theory, Experimental Techniques and Effect on Separations,” Electrophoresis, Vol. 25, No. 2, 2004, pp. 187-202. doi:10.1002/elps.200305754

[5] Z. Zhu, M. N. Toksöz and X. Zhan, "Experimental Studies of Streaming Potential and High Frequency Seismoelectric Conversion," Consortium Reports of MIT Earth Resources Laboratory, 2009.

[6] J. H. Saunders, M. D. Jackson and C. C. Pain, “A New Numerical Model of Electrokinetic Potential Response during Hydrocarbon Recovery," Geophysical Reserach Letters, Vol. 33, No. 15, 2006, pp. L15316, 1-6.

[7] J. Lyklema, "Fundamentals of Colloid and Interface Science,” Academic Press, London, 1993.
[8] T. Ishido and H. Mizutani, "Experimental and Theoretical Basis for Electrikinetic Phenomena in Rock-Water Systems and Its Application to Geophysis,” Journal of Geophysical Research, Vol. 86, 1981, pp. 1763-1775. doi:10.1029/JB086iB03p01763

[9] D. B. Pengra, S. X. Li and P. Wong, "Determination of Rock Properties by Low Frequency AC Electrokinetics," Journal of Geophysical Research, Vol. 104, No. B12, 1999, pp. 29485-29508. doi:10.1029/1999JB900277

[10] A. Revil, D. Hermitte, M. Voltz, R. Moussa, J.-G. Lacas, G. Bourrié and F. Troland, "Self-Potential Sygnals Associated with Variations of the Hydrolic Head during an Infiltration Experiment," Geophysical Research Letters, Vol. 29, No. 7, 2002. doi:10.1029/2001GLO14 294

[11] R. Burridge and J. B. Keller, "Poroelastisity Equations Derived from Microstructure," Journal of the Acoustical Society of America, Vol. 70, No. 4, 1981, pp. 1140-1146. doi:10.1121/1.386945

[12] E. Sanchez-Palencia, "Non-Homogeneous Media and Vibration Theory,” Springer, Heidelberg, 1980.

[13] S. R. Pride, A. F. Gangi and F. D. Morgan, "Deriving the Equations of Motion for Porous Isotropic Media," Journal of the Acoustical Society of America, Vol. 92, No. 6, 1992, pp. 3278-3290. doi: $10.1121 / 1.404178$

[14] S. R. Pride and J. G. Berryman, "Linear Dymanics of Double-Porosity Dual-Permeability Materials i. Governing Equations and Acoustic Attenuation," Physical Review E, Vol. 68, No. 3, 2003, p. 036603. doi:10.1103/PhysRevE.68.036603

[15] S. R. Pride, "Governing Equations for the Coupled Electromagnetics and Acoustics of Porous Media," Physical Review B, Vol. 50, No. 21, 1994, pp. 1567815696. doi:10.1103/PhysRevB.50.15678

[16] G. Allaire, "Homogenization and Two-Scale Convergence,” SIAM Journal on Mathematical Analysis, Vol. 23, No. 6, 1992, pp. 1482-1518. doi:10.1137/0523084

[17] A. Bensoussan, J. -L. Lions and G. Papanicolaou, “Asymptotic Analysis for Periodic Structures,” North-Holland, Amsterdam, 1978.

[18] L. Tartar, “An Introduction to Navier-Stokes Equation and Oceanography," Series: Lecture Notes of the Unione Matematica Italiana, Vol. 1, 2006.

[19] J. G. Berryman, "Comparison of Two Up-Scaling Methods in Poroelasticity and Its Generalizations," Proceedings of 17th ASCE Engineering Mechanics Conference, Newark, June 2004, pp. 13-16.

[20] A. N. Chatterjee, D. M. Cannon, E. N. Gatimu, J. V. Sweedler, N. R. Aluru and P. W. Bohn, "Modelling and Simulation of Ionic Currents in Three Dimensional Microfluidic Devices with Nanofluidic Interconnects," Journal of Nanopart. Research, Vol. 7, No. 4-5, 2005, pp. 507-516. doi:10.1007/s11051-005-5133-X

[21] Y. Amirat and V. Shelukhin, "Homogenization of Electroosmotic Flow Equations in Porous Media," Journal of Mathematical Analysis and Applications, Vol. 
342, No. 2, 2008, pp. 1227-1245. doi:10.1016/j.jmaa.2007.12.075

[22] Y. Amirat and V. Shelukhin, "Homogenization of the Poisson-Boltzmann Equation” In: A. V. Fursikov, G. P. Galdi and V. V. Pukhnachev Eds., Advances in Mathematical Fluid Mechanics, The Alexander V. Kazhikhov Memorial Volume, Birkhäuser Verlag, Basel, 2010, pp. 29-41,

[23] V. V. Shelukhin and Y. Amirat, "On Electrolytes Flow in Porous Media,” Applied Mathematics and Technical Physics, Vol. 49, No. 4, 2008, pp. 162-173.

[24] J. C. Maxwell, "Treatise on Electricity and Magnetism," Clarendon Press, Oxford, 1881.

[25] S. R. de Groot and P. G. Mazur, "Non-Equilibrium Thermodynamics,” North-Holland, Amsterdam, 1962.

[26] J. Neev and F. R. Yeatts, "Electrokinetic Effects in Fluid Saturated Poroelastic Media,” Physical Review B, Vol. 40, No. 13, 1989, pp. 409135-409141. doi:10.1103/PhysRevB.40.9135

[27] V. V. Kormiltsev and V. V. Ratushnyak, "Theoretical and Experimental Basis for Spontaneous Polarization of
Rocks in the Oil and Gas Wells," Ekaterinburg, URO RAS (in Russian), 2007.

[28] P. Sennet and J. P. Oliver, "Colloidal Dispersion, Electrokinetic Effects, and the Concept of the Zeta Potential; Chemistry and Physics of Interfaces,” American Chemical Society, Washington, D.C., 1965.

[29] V. V. Shelukhin and S. A. Terentev, "Frequency Dispersion of Dielectric Permittivity and Electric Conductivity of Rocks via Two-Scale Homogenization of the Maxwell Equations," Progress in Electromagnetic Research B, Vol. 14, 2009, pp. 175-202. doi:10.2528/PIERB09021804

[30] V. V. Shelukin and S. A. Terentev, "Homogenization of Maxwell Equations and the Maxwell-Wagner Dispersion,” Doklady Earth Sciences, Vol. 424, No. 3, 2009 , pp. 155-159. doi:10.1134/S1028334X09010334

[31] E. D. Shchukin, A. V. Pertsev and E. A. Amelina, "Colloidal Chemistry," Vysshaya Shkola, Moscow, 1992.

[32] B. Wurmstich and F. D. Morgan, "Modeling of Streaming Potential Responses Caused by Oil Pumping," Geophysics, Vol. 59, No. 1, 1994, pp. 46-56. doi:10.1190/1.1443533 